\title{
Combo optical soliton and rogue wave solutions of the time fractional perturbed Radhakrishnan-Kundu-Lakshmanan model
}

\author{
Md. Fazlul Hoque ${ }^{1}$, Fahad Sameer Alshammari ${ }^{2}$, and Harun-Or- Roshid ${ }^{1}$ \\ ${ }^{1}$ Pabna University of Science and Technology \\ ${ }^{2}$ Prince Sattam bin Abdulaziz University
}

June 20, 2020

\begin{abstract}
In this paper, we apply the $\$ \backslash \tan (\backslash$ circleddash $/ 2) \$$ expansion and the Kudryashov general approaches to the time fractional perturbed Radhakrishnan-Kundu -Lakshmanan (RKL) equation. These integration schemes provide a number of optical soliton solutions of the model. The solutions registered with constraint conditions on the parameters that follow their existence criteria. To the constraint conditions, the solutions offer various transmission signals through optical fibres, such as double periodic optical solitons, combo optical periodic and rogue waves, combo periodic and shock waves, combo periodic and solitons, and combo double singular solitons. Moreover, after interaction of rogue and periodic waves, it is shown that the rogue waves are going to diminish after a certain time keeping periodic nature of the interaction. In fact, interaction of periodic and rogue waves produces periodic rogue type breather waves, that indicates the amplitude of the rogue waves gradually decreases, and vanishes after a certain time. Some dynamical signals are plotted in the graphs by picking suitable values on the parameters.
\end{abstract}

\section{Hosted file}

RKLM.pdf available at https://authorea.com/users/335309/articles/461145-combo-opticalsoliton-and-rogue-wave-solutions-of-the-time-fractional-perturbed-radhakrishnan-kundulakshmanan-model 

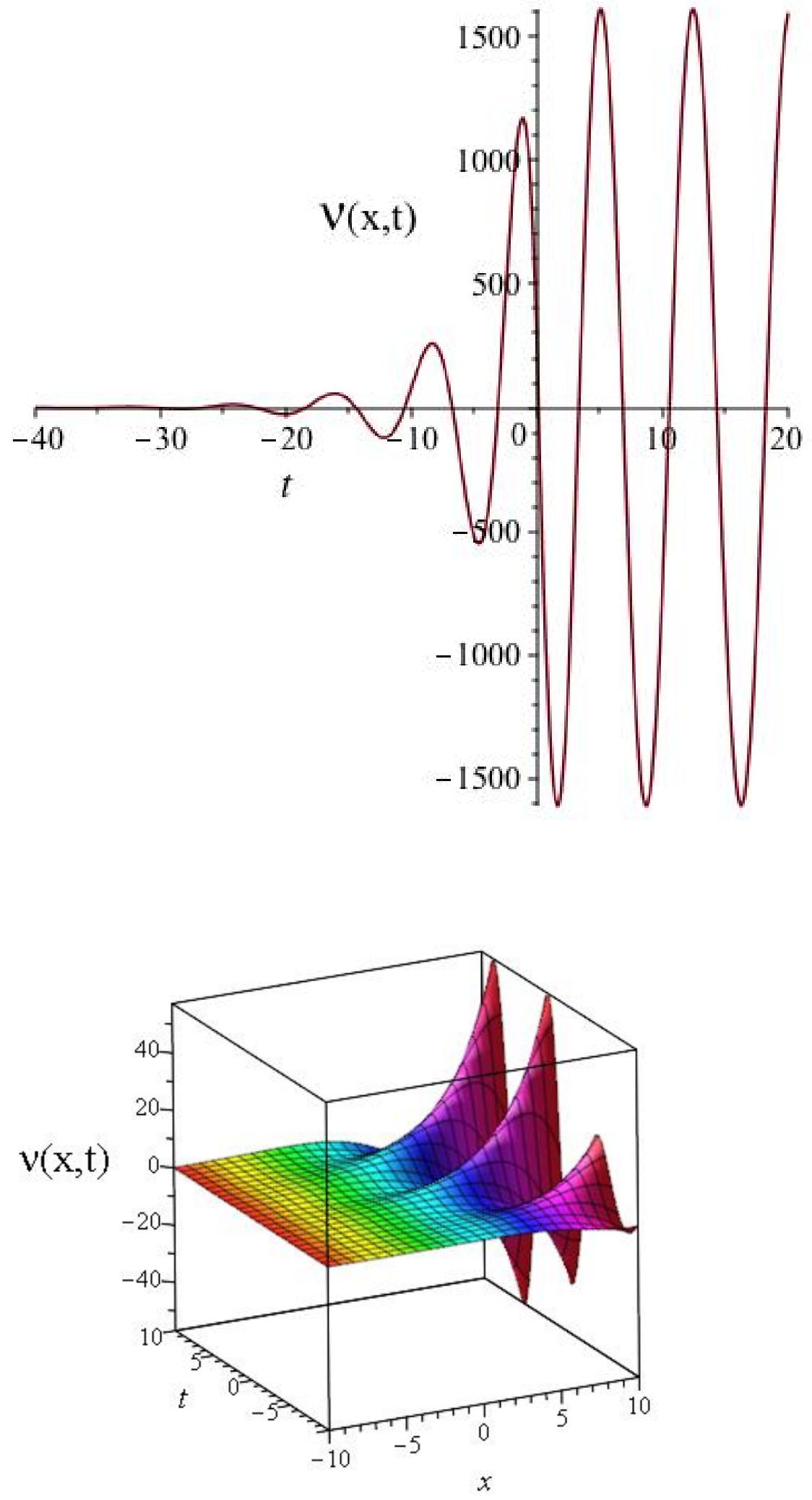

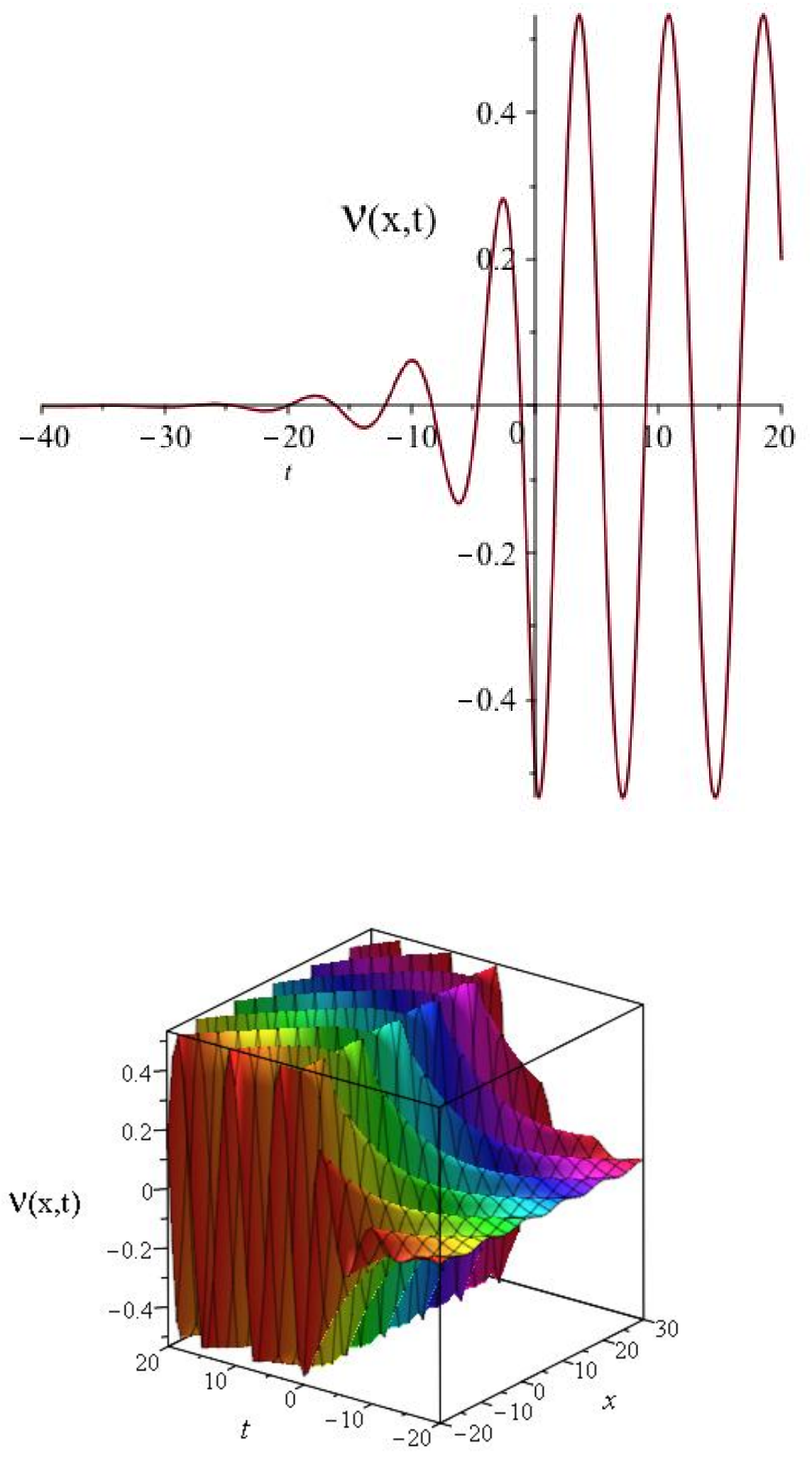

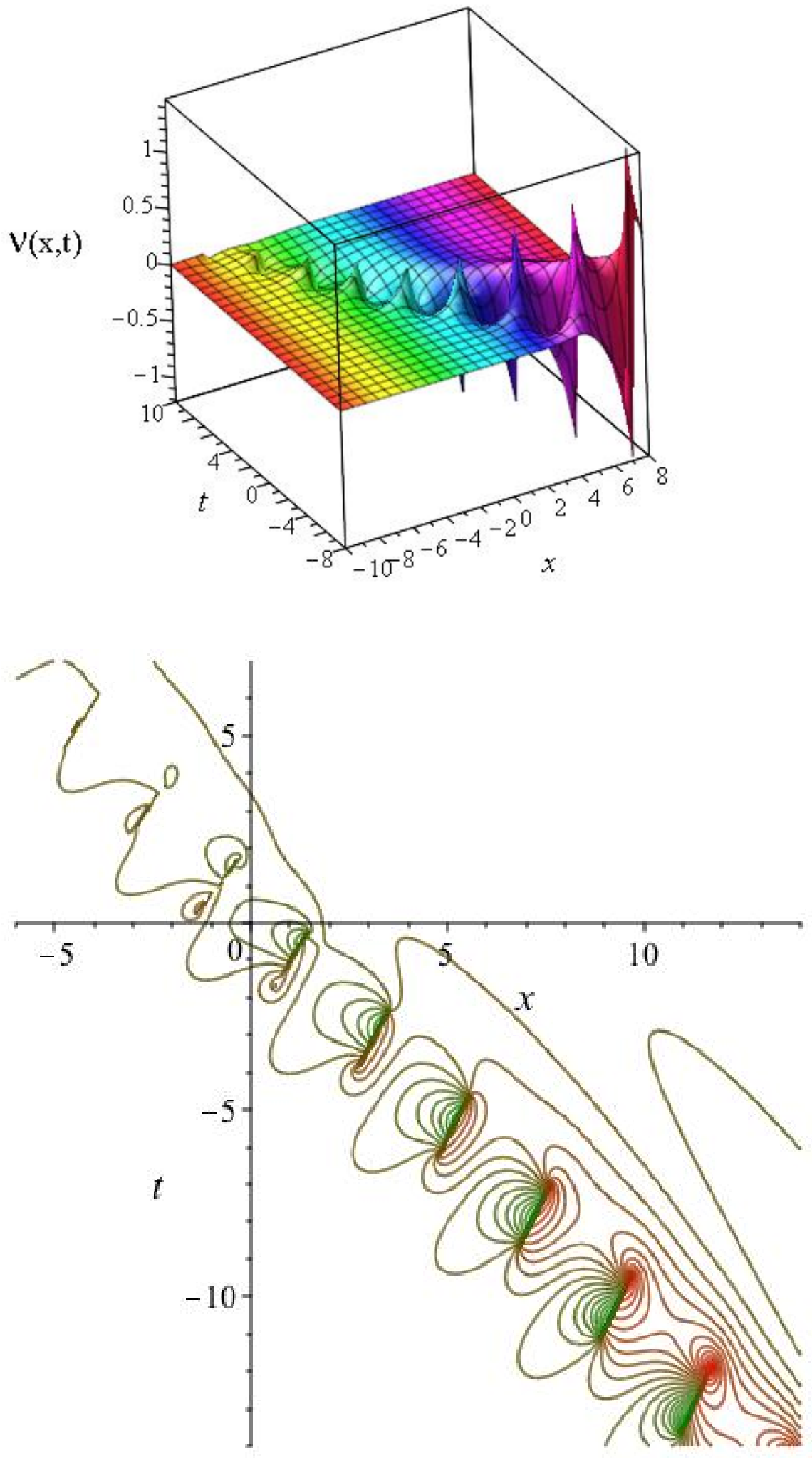

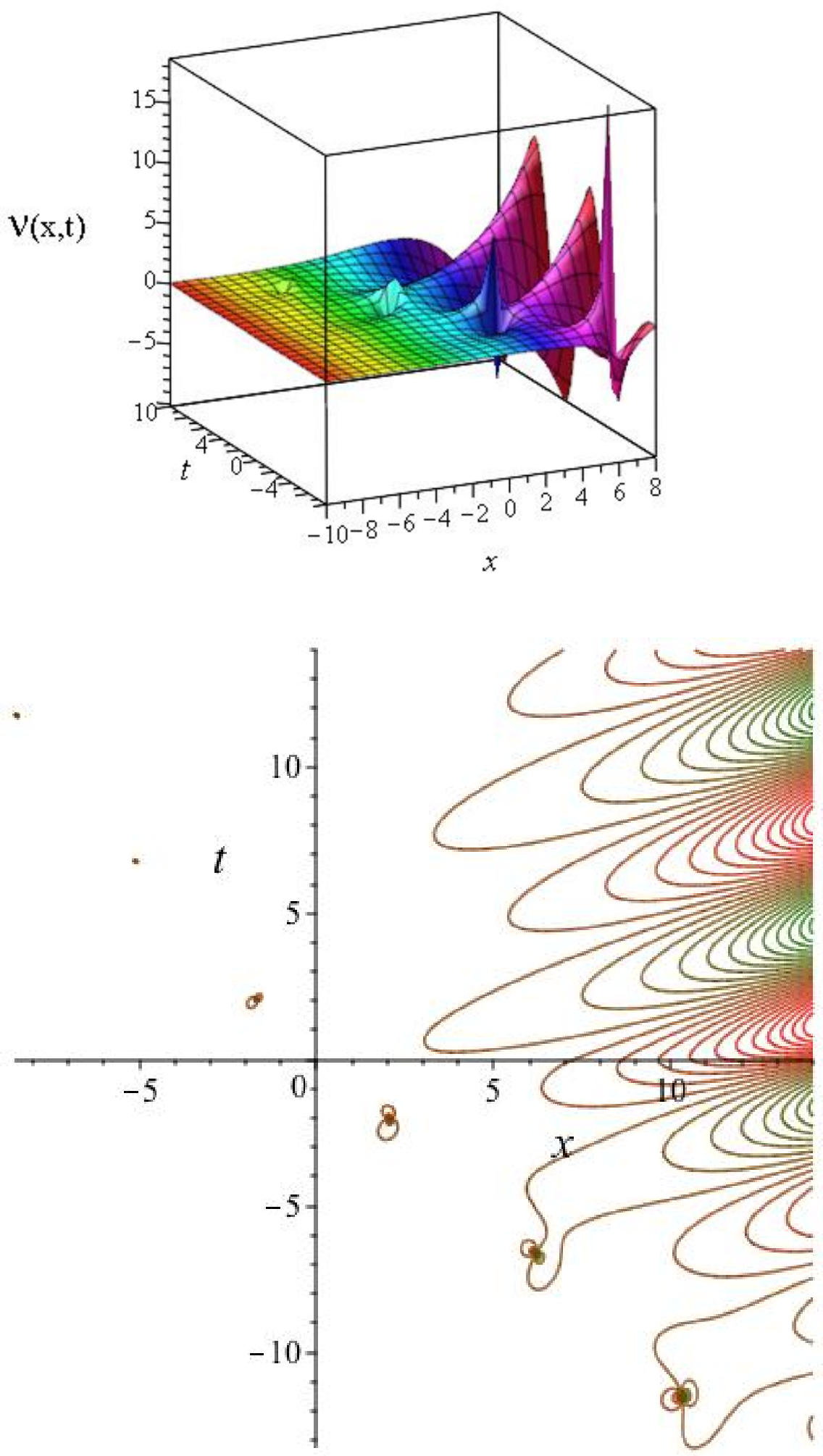

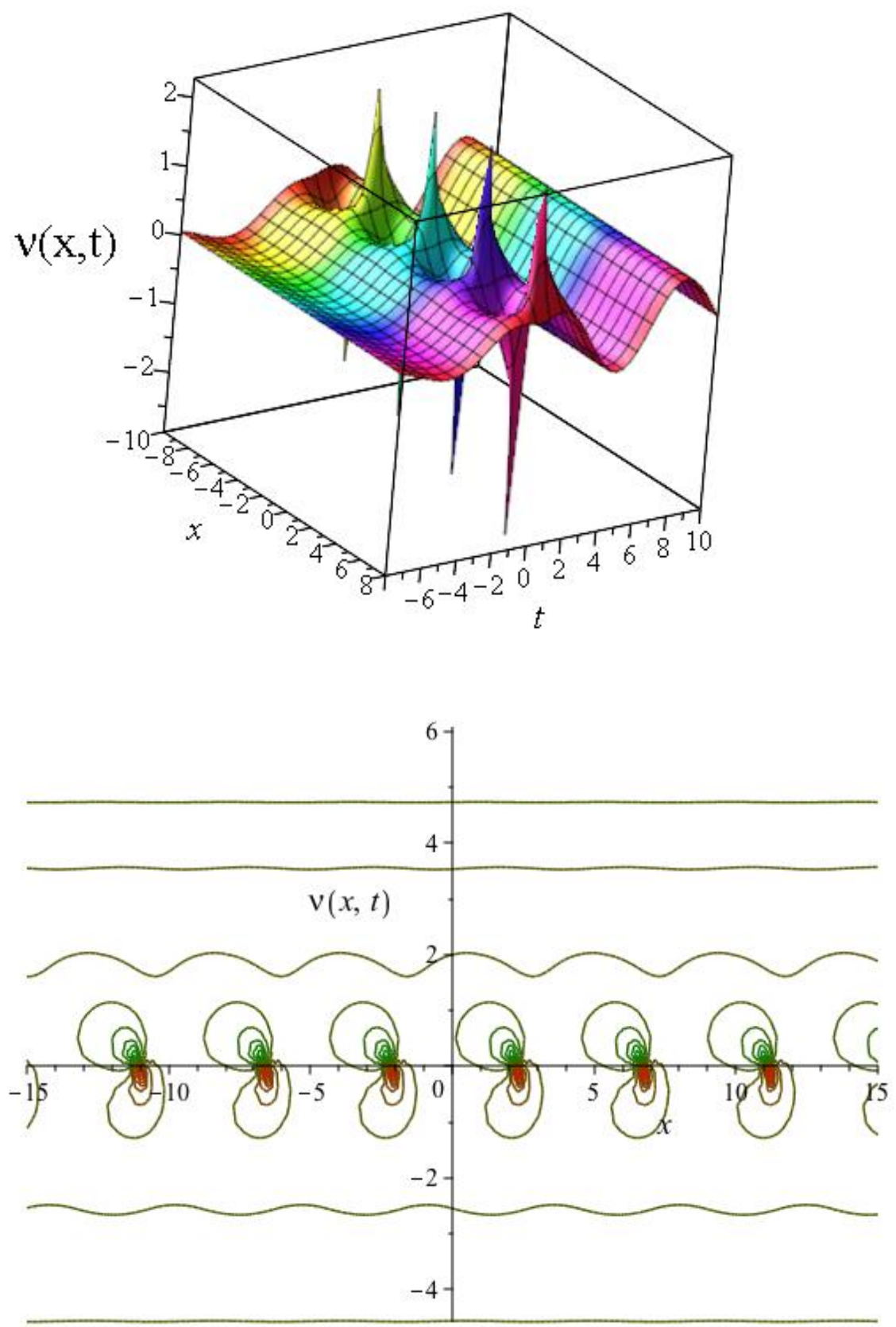

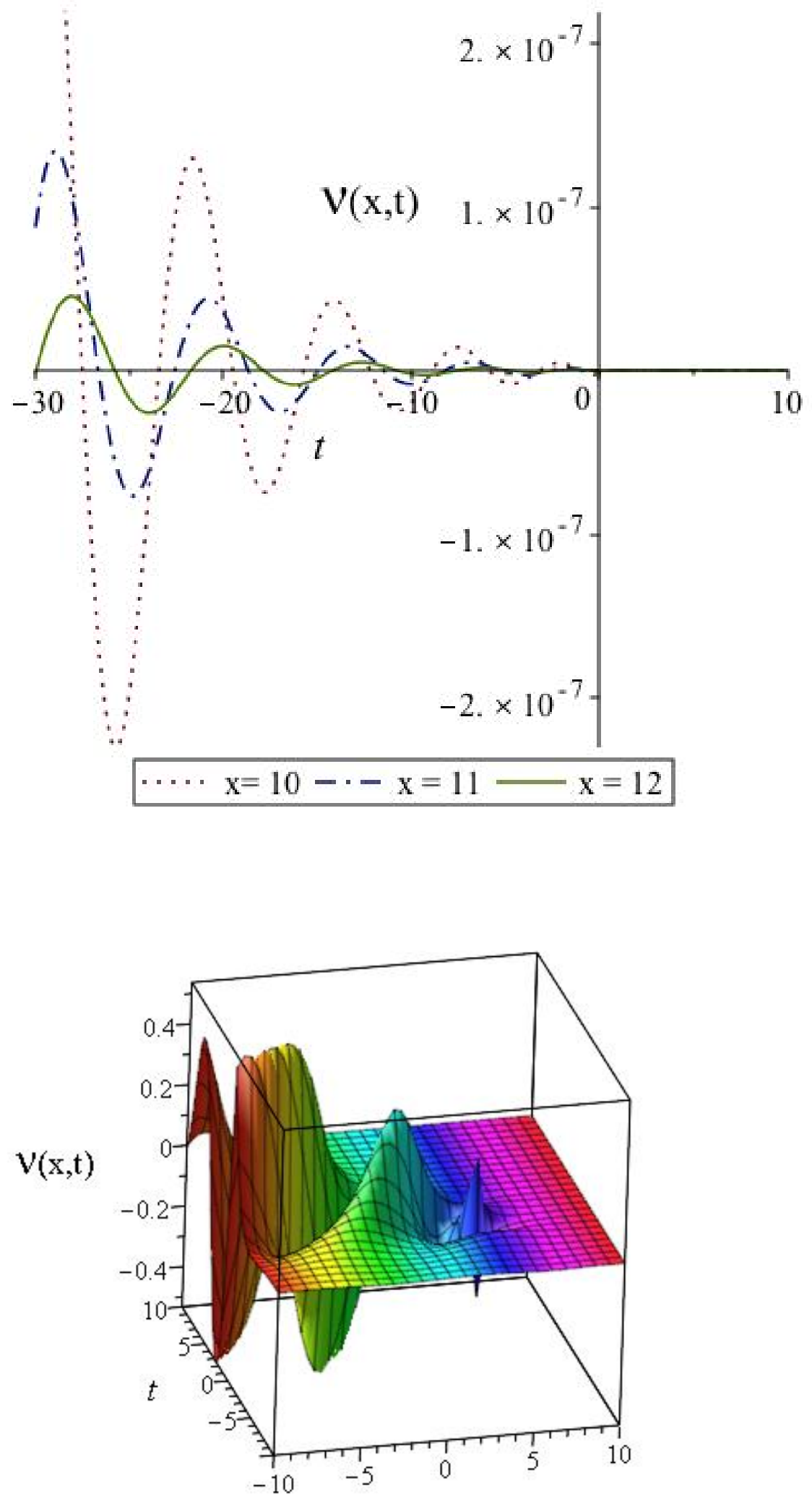

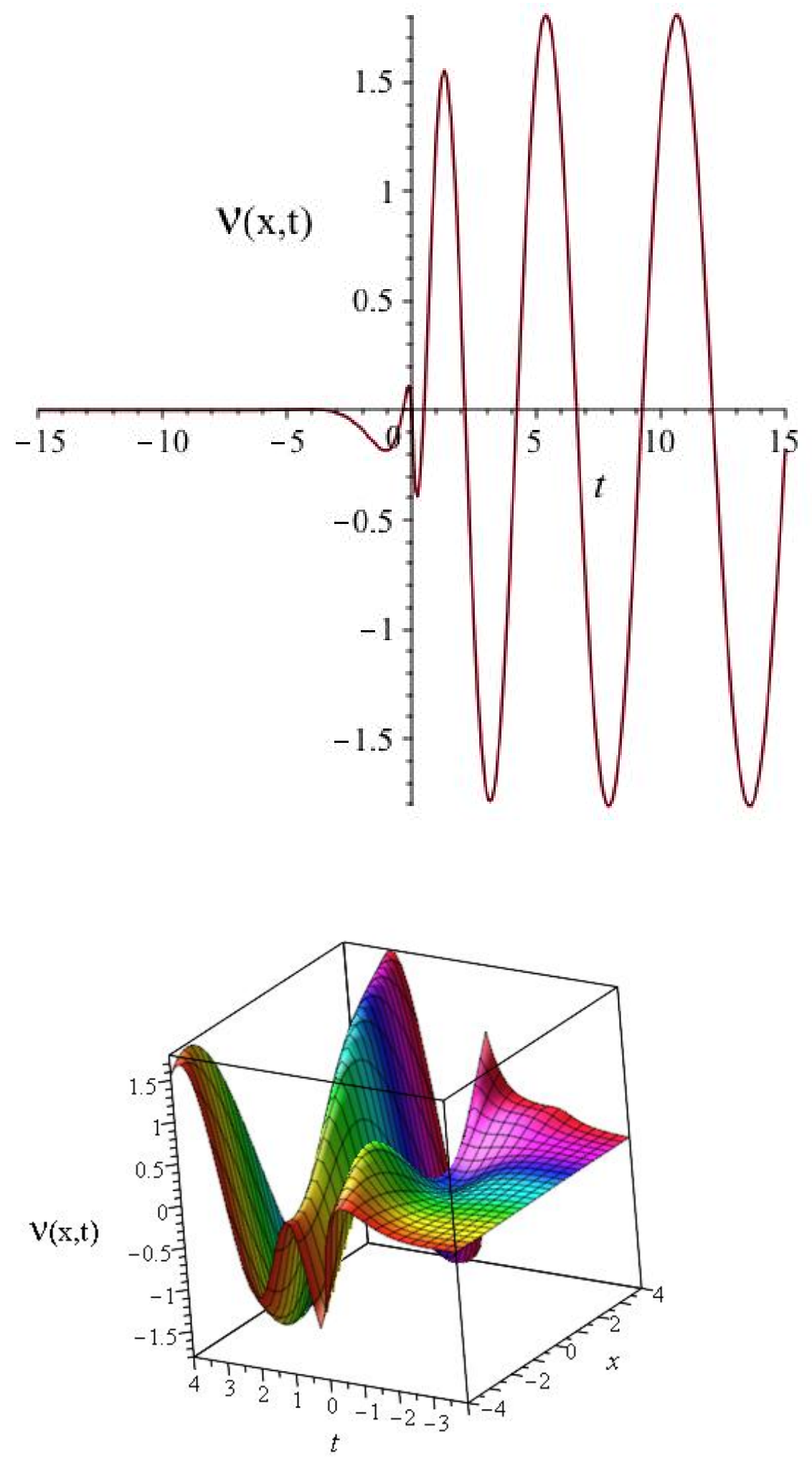

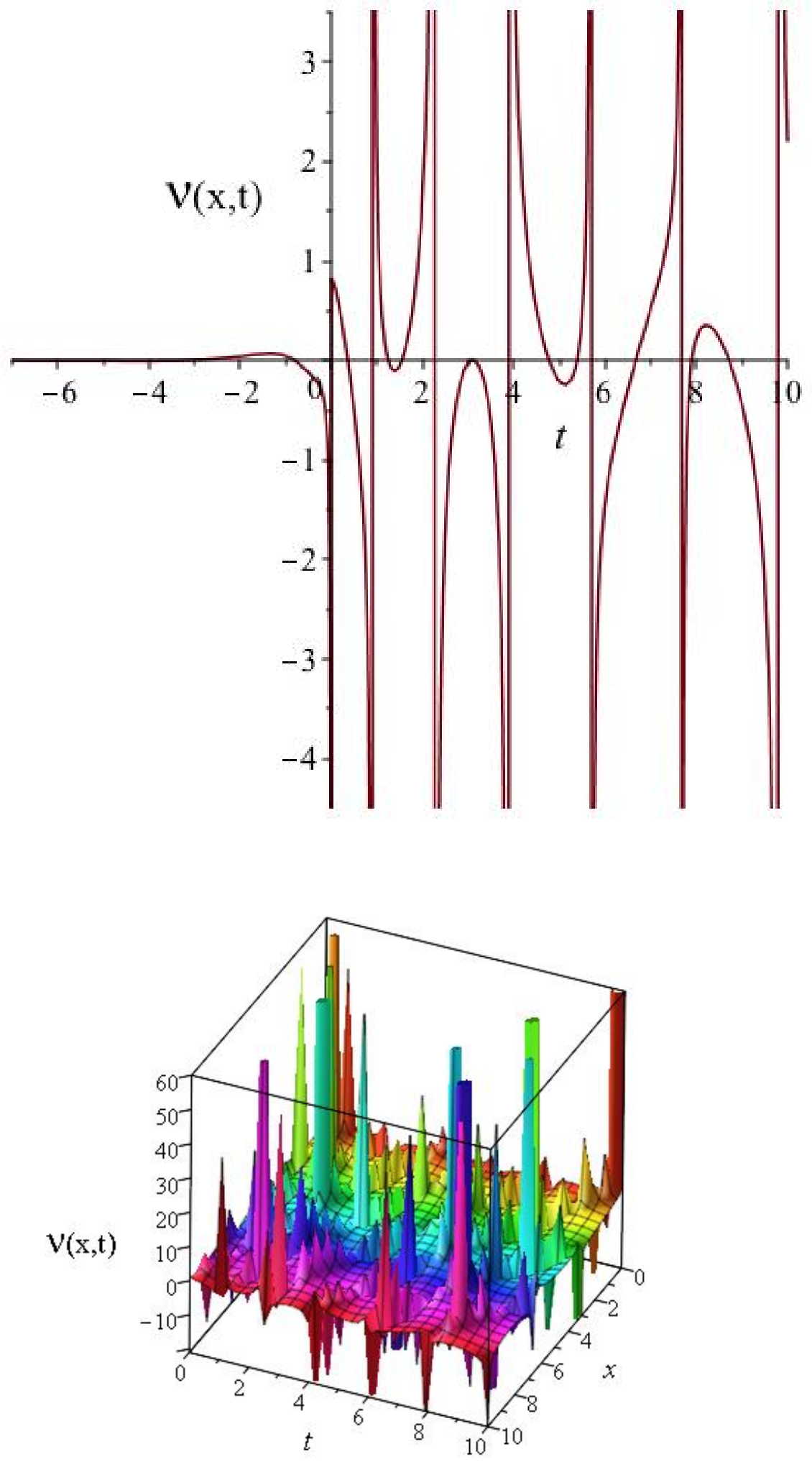